\title{
Primary Reconstruction is a Good Option in the Treatment of Urinary Fistula after Kidney Transplantation
}

\author{
Eduardo Mazzucchi, Guilherme L. Souza, Marcelo Hisano, Ioannis M. Antonopoulos, Affonso \\ C. Piovesan, William C. Nahas, Antonio M. Lucon, Miguel Srougi \\ Renal Transplantation Unit, Division of Urology, General Hospital, University of Sao Paulo \\ Medical School, SP, Brazil
}

\begin{abstract}
Objectives: urinary fistula is a morbid complication after renal transplantation leading to graft losses and patient death. We review and update our data on urinary fistula after renal transplantation and the outcome after surgical and conservative management.

Materials and Methods: the charts of 1046 renal transplants were reviewed. Transplants were performed through an extended inguinotomy; vascular anastomoses to the iliac vessels and urinary reconstruction accomplished through the Gregoir technique. Fistulae were diagnosed by urinary leaks through the incision or by the occurrence of a collection in the iliac fossa. Patient was treated surgically or conservatively according to the characteristics of the fistula and patient clinical status.

Results: Thirty one fistulae were diagnosed (2.9\%). Twenty nine leaks due to ureteral necrosis and 2 due to reimplantation fault. The incidence of leaks among cadaver and live donor transplants was 3.22\% and 2.63\%, respectively $(\mathrm{p}=0.73)$. Among diabetic and non diabetic patients the incidence of urinary leaks was $6.4 \%$ and $2.6 \%$, respectively $(\mathrm{p}=0.049)$. Treatment consisted in anastomosis of the graft ureter or pelvis with the ureter of the recipient in 17 cases with success in $13(76.5 \%)$. Prolonged bladder drainage was employed in 7 cases and the fistula healed in $4(57 \%)$. Ureteral reimplantation was performed in 3 cases and did not work in any of them. Ureteral ligature plus nephrostomy was employed in two cases and worked in one (50\%). Percutaneous nephrostomy and ureteral stenting with double $\mathrm{J}$ catheter were employed in one case each and worked in both.

Conclusions: The anastomosis of the graft ureter with the ureter of the recipient is a good method for treating urinary fistulae after renal transplantation when local and systemic conditions are good. Ureteral ligature associated to nephrostomy should be applied in cases of unfavorable local conditions or clinically unstable patients.
\end{abstract}

Key words: renal transplantation; urinary fistula; treatment outcome

Int Braz J Urol. 2006; 32: 398-404

\section{INTRODUCTION}

Since the beginning of renal transplant era, urological complications have remained an important source of morbidity and mortality among transplanted patients. Among urological complications, urinary leaks, ureteral obstruction and vesico-ureteral reflux are the most frequent ones. Urinary fistula still leads to graft losses and deaths in the modern transplant era. Its incidence has fallen in the last decades and is nowadays between 2 and 5\% (1-3). The morbidity and mortality also decreased significantly from the 
early 80 's due to an improvement in immunosuppression protocols using low dose steroids, prompt diagnosis and better surgical treatment. Mortality is now around $8 \%$ (4). The number of renal transplant has increased drastically in our country in the last five years and the real incidence, the main causes and outcome of patients with this complication after treatment is unknown. Herein we update our data about urinary fistulae as well the treatment employed and its results.

\section{MATERIALS AND METHODS}

The charts of patients transplanted between January 1994 and December 2003 were reviewed. A total of 1046 renal transplants were performed during the study period; 456 grafts $(43.6 \%)$ were obtained from live donors and 590 (56.4\%) from cadavers. Nephrectomy in live donors was performed either laparoscopically or by classic lumbotomy. Cadaveric kidneys were procured by laparotomy and in situ perfusion with Wisconsin or Eurocollins solutions were employed in all cases.

Renal transplants were performed by the usual technique; the access to the retroperitoneum was obtained by an extended inguinotomy. Vascular anastomoses were to the iliac vessels or exceptionally to the inferior vena cava. Urinary reconstruction was accomplished by ureteroneocystostomy (Gregoir technique) in the majority of the cases. A Foley catheter was used to drain the bladder for 5 days after transplant. Double J stents were used exceptionally when ureteral or bladder condition was poor. Drains after transplant were used only if bleeding was present at the end of the surgery.

Immunosuppression was based in a triple regimen: cyclosporine or rapamycin, azathioprine or micophenolate mofetil and prednisone. Induction with Okt3 (ortoclone), antithimocyte globulin (ATG) or anti IL-2 (Daclizumab) were used in re-transplants or in patients with a high panel reactive antibody. Acute rejection episodes were treated by corticosteroids or with immunoglobulins.

Urinary leaks were diagnosed by the occurrence of urine extravasations through the surgical wound, or in the presence of a fluid collection at the iliac fossa, by the occurrence of intense dysuria or polaciuria or still, after investigation of unexplained graft dysfunction. Ultrasound was the initial diagnostic method employed but the diagnosis was confirmed by cystogram and by fluid analysis when a collection was present. In some cases a tomography with injection of iodinated contrast through the Foley catheter was performed.

Surgical treatment was performed in all patients except those presenting with minimal extravasation at the ureteral reimplantation site and clinically stable. This group was initially treated by urinary drainage. In cases of unfavorable outcome after clinical treatment, surgery was indicated. Surgery was the initial aproach for big extravasation or when leaks arising from the mid or upper ureter were suspected. We used the same incision of the transplant to access the fistulae. The type of surgical reconstruction was based on the intraoperative evaluation of the extent of the ureteral necrosis and local and systemic condition of the patient at the time of surgery. Primary reconstruction with the ureter of the recipient or a new ureteral reimplantation were performed preferentially when local and systemic condition allowed; if local or systemic infection were present and the patient was clinically unstable an ureteral ligature associated to a nephrostomy was performed. Ureteral stenting alone was used exceptionally. All patients received prophylactic or therapeutic antibiotic according to the antibiogram of the collected fluid.

Statistical analysis of the data was performed using Fischer exact test, with $p<0.05$ considered significant.

\section{RESULTS}

One thousand and forty six renal transplants were performed during the study period; 456 grafts $(43.6 \%)$ were obtained from live donors and 590 $(56.4 \%)$ from cadavers. Mean cold ischemia time was 52 minutes for live donor kidneys (range 30-105 minutes) and 18 hours for cadaver donor kidneys (range 10-28 hours). Follow-up ranged from 21 to 118 
months (mean 67.3 months).Thirty one urinary leaks were diagnosed $(2.9 \%)$. Nineteen leaks $(61.3 \%)$ occurred among cadaver donor grafts and 12 (38.7\%) among live donor grafts. The incidence of leaks among cadaver transplants was $3.22 \%$ and among live donor transplants was $2.63 \%(\mathrm{p}=0.73)$. Fistulae were diagnosed between the first and the 131 postoperative day (mean 28 post operative day). Thirteen fistulae $(42 \%)$ were diagnosed between the first and the 15th postoperative day and $25(80 \%)$ during the first month after transplant; patients' characteristics are in Table-1.

In 4 of the cases $(12.9 \%)$ an inferior polar artery was present in the graft and was preserved or reconstructed. One patient had an ileal bladder augmentation and another one had a continent reservoir with a Mitrofanoff catheterization mechanism. Ninety three patients were diabetic at the time of renal transplantation (8.9\%); 6 developed urinary fistulae $(6.4 \%)$ compared to 25 non diabetic patients $(2.6 \%)$ ( $\mathrm{p}=$ 0.049). Double-J stents were used in 38 transplants; urinary leaks were observed in 2 of these patients $(5.2 \%)$. On the other hand urinary leaks occurred in 29 patients where double J were not used $(2.9 \%)(\mathrm{p}=$ $0.319)$.

Among 456 grafts from live donors 48 $(10.5 \%)$ were obtained by laparoscopy; 2 developed urinary fistula $(4.1 \%) ; 10$ fistulae were found among 408 open nephrectomy donors $(2.4 \%)(p=0.36)$.
Fistulae were caused by ischemic ureteral necrosis in 29 cases $(93.5 \%)$ and by reimplantation fault in 2 patients.

Treatment consisted in uretero-ureteric anastomosis with the ureter of the recipient in 17 cases; in 14 cases a termino-lateral anastomosis was performed and in 3 patients graft ureter was anastomosed termino-terminally to the ureter of the recipient. A double-J catheter was used in all patients. Other forms of treatment comprised bladder drainage through prolonged catheterization in seven cases, a new ureteroneocystostomy over a double-J catheter in three cases, percutaneous nephrostomy in two cases, ureteral ligature plus nephrostomy and ureteral stenting with double-J catheter in one case each.

Resolution of the fistulae with one procedure was accomplished in 20 cases (64.5\%). Ureteral anastomosis with the ureter of the recipient was effective as an unique procedure in 13 cases (76.4\%), bladder drainage in $4(57 \%)$, percutaneous nephrostomy in 1 $(50 \%)$, ureteral ligature plus nephrostomy and double $\mathrm{J}$ stenting in one case each (100\%) and ureteroneocystostomy did not work in any of the 3 cases where they were employed (Table-2).

Eleven failures occurred and were treated surgically; treatment options comprised new anastomosis with the ureter of the recipient (6 cases), ligature of the ureter associated to a nephrostomy (2 cases), double $\mathrm{J}$ stenting (one case), ureter

Table 1 - Characteristics of transplanted patients with urinary fistula.

\begin{tabular}{ll}
\hline Patients & 31
\end{tabular}

Male / Female

Age - range / mean / median (years)

Period of dialysis- range / mean (months)

Primary renal disease
31

$19 / 12$

$14-63$ / $40.6 / 40$

2-184 / 55.4

chronic glomerulonephritis $=8$

diabetes mellitus $=6$

systemic arterial hypertension $=4$

vesicoureteral reflux / chronic pyelonephritis $=4$

systemic lupus eritematous $=3$

focal segmental glomerulosclerosis $=3$

membranoproliferative glomerulonephritis $=1$

amiloidosis $=1$

Ig A nephropathy = 1

19 / 12 
Table 2 - Treatment employed in urinary fistula.

\begin{tabular}{lrrrr}
\hline First Treatment & N & \% & Resolution \\
& & & N & \% \\
\hline Uretero-ureterostomy with ureter of recipient & 17 & 54.8 & 13 & 76.5 \\
Bladder drainage & 7 & 25.8 & 4 & 57 \\
Ureteral reimplantation & 3 & 9.7 & & zero \\
Percutaneous nephrostomy & 2 & 6.4 & 1 & 50 \\
Ureteral ligature + nephrostomy & 1 & 3.2 & 1 & 100 \\
Ureteral ligature + double J stenting & 1 & 3.2 & 1 & 100 \\
Total & 31 & 100 & 20 & 64.5 \\
\hline
\end{tabular}

reimplantation (one case) and nephrectomy. Additionally a nephrectomy of a thrombosed kidney was performed. Three cases needed a third procedure to heal the fistula. This way, $64.5 \%$ of the urinary fistulae were resolved with one procedure, $90 \%$ with two procedures and $10 \%$ needed more than two procedures to heal.

One patient lost his graft due to venous thrombosis after the surgery for correction of a urinary fistula; 6 other patients lost their kidneys during follow- up: 5 due to chronic nephropathy and one due to humoral rejection. At the end of the study period, 17 kidneys were functioning (55\%).

Five patients died during the study period (16.1\%). Two patients died due to infectious complications and sepsis $(6.4 \%)$ directly related to the urinary leakage or due to its treatment; three other patients died of non related causes (one from a laryngeal tumor, one from pneumonia and one from cerebral ischemia).

\section{DISCUSSION}

Thanks to government policy the number of solid organs transplants experienced an expressive increase in our country in the last five years. This fact has also provoked an increase in the number of procurement and transplantation teams. Urinary fistula is not any more a frequent problem but continues to occur and may represent a morbid complication. Urinary fistulae arise frequently due to technical problems, during procurement or transplant surgery. Ure- teral necrosis due to ischemia is the most frequent cause and can be occasioned during organ retrieval or implantation when extensive ureteral dissection lead to blood supply damage and consequently to organ necrosis $(2,4-6)$. Ureteral necrosis was also the most frequent cause of fistulae in our casuistic, responding to $93.5 \%$ of all cases. Several other risk factors for the development of urinary fistulae have been mentioned: recipient's age, number of renal arteries, site of arterial anastomosis, type of donor, the occurrence of acute rejection episodes, type of urinary reconstruction, bladder problems, immunosuppressive regimen (4,7-10). Among them, recipients age $<10$ years, use of uretero-ureteric anastomosis and the use of high dose steroids are considered independent risk factors (7). In this series, leaks were more frequent among cadaver donor recipients and significantly more common among diabetic patients at the time of transplantation. Laparoscopic nephrectomy did not increase significantly the occurrence of urinary leaks.

Extravesical ureteroneocystostomy has proved to be easier and with a low incidence of complications $(8,11)$. Routine ureteral stenting does not reduce significantly the incidence of urinary fistulae and its use is recommended only in special cases (contracted bladder, difficult anastomosis) $(8,11)$. In our center the modified Gregoir technique has been the procedure of choice in the last 35 years and the incidence of ureteral complications has been low.

Early aggressive management is the key to prevent graft losses and reduces mortality $(3,4)$. Many options for urinary fistula management have been described with different rates of success: ureteral liga- 
ture and nephrostomy, ureteroureterostomy, pyeloureterostomy, ureteroneocystostomy, percutaneous nephrostomy associated to ureteral stenting, prolonged vesical drainage $(4,7-9,11)$. Percutaneous techniques like nephrostomy associated to antegrade ureteral stenting works in $40 \%$ of a much selected group of patients presenting with small fistulae from the distal ureter (8). Prolonged bladder drainage is indicated for small fistulae arising from the reimplantation site in clinically stable patients and in our hands a $50 \%$ of good results was obtained. Early open surgery is our preferred approach. Our policy is to perform primary urinary tract reconstruction whenever local and systemic condition allows. Termino-lateral anastomosis of the graft ureter or pelvis with the ureter of the recipient has been our preferred technique for the correction of urinary leaks; we obtained $77 \%$ success with this method, compatible with the results published in other studies $(11,12)$. Some groups use termino-terminal anastomosis with the ureter of the recipient (11) with good results but we observed two cases ureterohydronephrosis of the native kidney after ligation of the ureter for reconstruction after renal transplantation who required nephrectomy. Ureteroneocystostomy is used for reimplantation defects or for small distal ureteral necrosis and in our hands failed in all cases due to necrosis extension or incomplete ureteral and bladder wall resection during surgery. Although it did not work in this series, ureteral reimplantation remains an important option for urinary fistulae management. Ureteral ligature and nephrostomy is performed when there is gross infection of the fossa or when the patient presents in sepsis. According to this policy, a $65 \%$ of resolution was obtained with one procedure and $90 \%$ with 2 procedures. Ten patients needed a second procedure due to recurrence of the leakage. Recurrences were due to insufficient ureter resection, leaving an ischemic stump extension of the process after the surgery or inadequate anastomosis. We always leave a double $\mathbf{J}$ stent in these cases in order to reduce recurrences but stents do not work if the necrosis extends. Recurrences were always managed surgically and an anastomosis with the ureter of the recipient was the first choice. In one case the local condition was unfavorable and ureteral ligature with nephrostomy was performed.
Some patients needed a third procedure due to a new recurrence showing that the necrosis can extend after surgery and that extensive resection of the ureter is frequently necessary. Additionally we should say that it was not the aim of this paper to compare all the techniques employed for treating urinary leaks in this group of patients because this is a retrospective study, not designed for this kind of comparison; overall, this is an heterogeneous group of patients.

Mortality directly related to the fistula or to its correction was high in the early transplantation era (13) and nowadays is reported to range from 0 to $8 \%$ (11); in our series it was $6.4 \%$. These better results are due to an earlier and more aggressive approach, reduction in the amount of corticosteroids in the immunosuppressive regimen and to better antibiotics and clinical support. The increase in the experience with these cases can still improve such results.

\section{CONCLUSIONS}

In this retrospective evaluation urinary fistulae were more common among cadaver donor and diabetic recipients. The use double $\mathbf{J}$ stents did not reduce significantly the incidence of urinary leaks. Primary anastomosis of the graft ureter or pelvis to the ureter of the recipient was a good method for treating urinary fistulae when local and systemic conditions were good. Ureteral ligature associated to nephrostomy can be used when local or patient clinical condition is unfavorable.

\section{CONFLICT OF INTEREST}

None declared.

\section{REFERENCES}

1. Rigg KM, Proud G, Taylor RM: Urological complications following renal transplantation. A study of 1016 consecutive transplants from a single centre. Transpl Int. 1994; 7: 120-6. 
2. Shoskes DA, Hanbury D, Cranston D, Morris PJ: Urological complications in 1,000 consecutive renal transplant recipients. J Urol. 1995; 153: 18-21.

3. Nane I, Kadioglu TC, Tefekli A, Kocak T, Ander H, Koksal T: Urologic complications of extravesical ureteroneocystostomy in renal transplantation from living related donors. Urol Int. 2000; 64: 27-30.

4. Waltzer WC, Frischer Z, Shabtai M, Jao S, Rapaport FT: Early progressive management for the prevention of renal allograft loss and patient mortality following major urologic complications. Clin Transplantation 1992; 6: 318-322.

5. Dreikorn K: Problems of the distal ureter in renal transplantation. Urol Int. 1992; 49: 76-89.

6. Streeter EH, Little DM, Cranston DW, Morris PJ: The urological complications of renal transplantation: a series of 1535 patients. BJU Int. 2002; 90: 627-34.

7. Gibbons WS, Barry JM, Hefty TR: Complications following unstented parallel incision extravesical ureteroneocystostomy in 1,000 kidney transplants. J Urol. 1992; 148: 38-40.

\section{Correspondence address:}

Dr. Eduardo Mazzucchi

Rua Barata Ribeiro, 490 / 25

São Paulo,SP, 01308-000, Brazil

E-mail:mazuchi@terra.com.br

\section{EDITORIAL COMMENT}

The incidence of urological complications after kidney transplantation varies from $3 \%$ to $14 \%$, with a probable loss of the graft in $10 \%$ to $15 \%$ of cases and a mortality rate of up to $15 \%$, despite improvements in prevention, diagnosis, and treatment as well as the use of new immunosuppressive thera-
8. Campbell SC, Streem SB, Zelch M, Hodge E, Novick AC: Percutaneous management of transplant ureteral fistulas: patient selection and long-term results. J Urol. 1993; 150: 1115-7.

9. El-Mekresh M, Osman Y, Ali-El-Dein B, El-Diasty T, Ghoneim MA: Urological complications after living-donor renal transplantation. BJU Int. 2001; 87: 295-306.

10. Dominguez J, Clase CM, Mahalati K, MacDonald AS, McAlister VC, Belitsky P, et al.: Is routine ureteric stenting needed in kidney transplantation? A randomized trial. Transplantation. 2000; 70: 597-601.

11. Salomon L, Saporta F, Amsellem D, Hozneck A, Colombel M, Patard JJ, et al.: Results of pyeloureterostomy after ureterovesical anastomosis complications in renal transplantation. Urology. 1999; 53: 908-12.

12. Ghasemian SM, Guleria AS, Khawand NY, Light JA: Diagnosis and management of the urologic complications of renal transplantation. Clin Transplant. 1996; 10: $218-23$.

13. Dreikorn K: Problems of the distal ureter in renal transplantation. Urol Int. 1992; 49: 76-89.

Accepted after revision: March 20, 2006

pies (1). Urinary fistulae are considered early complications of transplantation. They occur in 3-5\% of cases in which no double-J stent has been used (2). Preservation of accessory arteries to the lower portion of the kidney is important, as they may constitute the blood supply of this segment of the collect- 
ing system or ureter. Their ligation may lead to necrosis and urinary fistulae $(1,2)$. Urinary fistulae can occur also on bladder or calyx due to a closure that is not watertight or to necrosis by ligature of a polar artery respectively (2). Furthermore, European Urology Guidelines on Renal Transplantation Committee recommends to use a short ureter and keep the periureteral fat around the hilus and ureteral vessels and to insert a prophylactic double J-stent and a bladder catheter (2). With regards to treatment, ureteral fistulae can be treated by open surgery or by percutaneous approach. In open surgery option, the ureter is re-cut and a double-J stented, uretero-ureteral anastomosis is made using the patient's original ureter. In case of percutaneous treatment, where it is possible to localize the fistula, it is worth trying nephrostomy and/or bladder catheter and double-J stent. Bladder fistulae can be treated by suprapubic or transurethral catheter. Caliceal fistulae are treated by nephrostomy or bladder catheter and double-J-stent. If this fails, polar nephrectomy can be tried.

The authors retrospectively report their experience and the data about urinary fistulas and the outcome after surgical and conservative management. They report to employ ureteral stent exceptionally at the surgeon discretion during transplantation. In the authors' opinion, routine ureteral stenting does not reduce significantly the incidence of urinary fistulas and its use is recommended only in special cases.
In our experience, although the double-J stent appeared to us to be a valid aid to guarantee the anastomosis patency in the first days immediately after the transplantation, it did not play a definite role.

Nevertheless, in a recent review Wilson et al. (3) performed a meta-analysis to examine the benefits and harms of routine ureteric stenting to prevent urological complications in kidney transplant recipients. The results of this review suggest that prophylactic stenting reduces the incidence of major urological complications and should be recommended on the basis of currently available randomized controlled trials (3).

\section{REFERENCES}

1. Li Marzi V, Filocamo MT, Dattolo E, Zanazzi M, Paoletti MC, Marzocco M, et al.: The treatment of fistulae and ureteral stenosis after kidney transplantation. Transplant Proc. 2005; 37: 2516-7.

2. Kalble T, Lucan M, Nicita G, Sells R, Burgos Revilla FJ, Wiesel M: European Association of Urology. Guidelines on renal transplantation. 2004. (http:// www.uroweb.nl/files/uploaded_files/guidelines/ 22891_Renal_Transplant.pdf)

3. Wilson CH, Bhatti AA, Rix DA, Manas DM: Routine intraoperative stenting for renal transplant recipients. Transplantation. 2005; 80: 877-82.

Dr. Vincenzo Li Marzi Dr. Maria Teresa Filocamo Dr. Giulio Nicita Department of Urology II, University of Florence Florence, Italy E-mail:vlimarzi@hotmail.com

Dr. Maria Zanazzi Department of Nephrology \& Transplantation Careggi Hospital, Florence, Italy 